Instituto Internacional de Investigación y Desarrollo Tecnológico Educativo INDTEC, C.A. DOI: https://doi.org/10.29394/Scientific.issn.2542-2987.2019.4.E.10.173-187

OAI-PMH: http://www.indteca.com/ojs/index.php/Revista Scientific/oai

Artículo Original / Original Article

\title{
Impacto de las nuevas tecnologías en la masificación de la educación
}

\author{
Autor: Francisco Javier Calderón Pujadas \\ Universidad Miguel de Cervantes, UMC \\ fcalderon@umcervantes.cl \\ Talca, Chile

$\frac{\text { fcalderon@umcervantes.cl }}{\text { Talca, Chile }}$
https://orcid.org/0000-0003-4003-4849

\section{Resumen}

En el mundo de hoy juega un papel determinante la tecnología de la información y las comunicaciones. La nueva era industrial tiene como característica principal el consumo de información. Metodológicamente se ubicó en un estudio con un diseño no experimental, tipo de investigación de campo y nivel descriptivo, con una población de 20 docentes y 40 estudiantes de Proyecto de Investigación a nivel de Maestría de la Universidad Valle de Momboy, siendo la muestra censal igual a la población. Para obtener la información, se empleó la técnica de la encuesta, el instrumento fue el cuestionario, con dieciséis (16). Se sometió a su validez a través del juicio de experto y la confiabilidad calculada fue de 0,794, según el Alfa de Cronbach, se recogió la información, para realizar los cálculos estadísticos y se analizaron los resultados, lo que permitió realizar un diagnóstico de la problemática El surgimiento de este nuevo modelo de sociedad introduce cambios en los esquemas económicos y sociales, con una tendencia a simplificar los complejos procesos de producción y de manejo de la información. La sociedad del futuro nos plantea retos interesantes en los aspectos de la vida cotidiana, girando todo este alrededor de un aspecto en común: la tecnología.

Palabras clave: tecnología; educación; información.

Cómo citar este artículo:

Calderón, F. (2019). Impacto de las nuevas tecnologías en la masificación de la educación. Revista Scientific, 4(Ed. Esp.), 173-187, e-ISSN: 2542-2987. Recuperado de: https://doi.org/10.29394/Scientific.issn.2542-2987.2019.4.E.10.173-187

Fecha de Recepción:

11-05-2019
Fecha de Aceptación:

18-07-2019
Fecha de Publicación: 05-09-2019 


\section{Impact of new technologies on the massification of education}

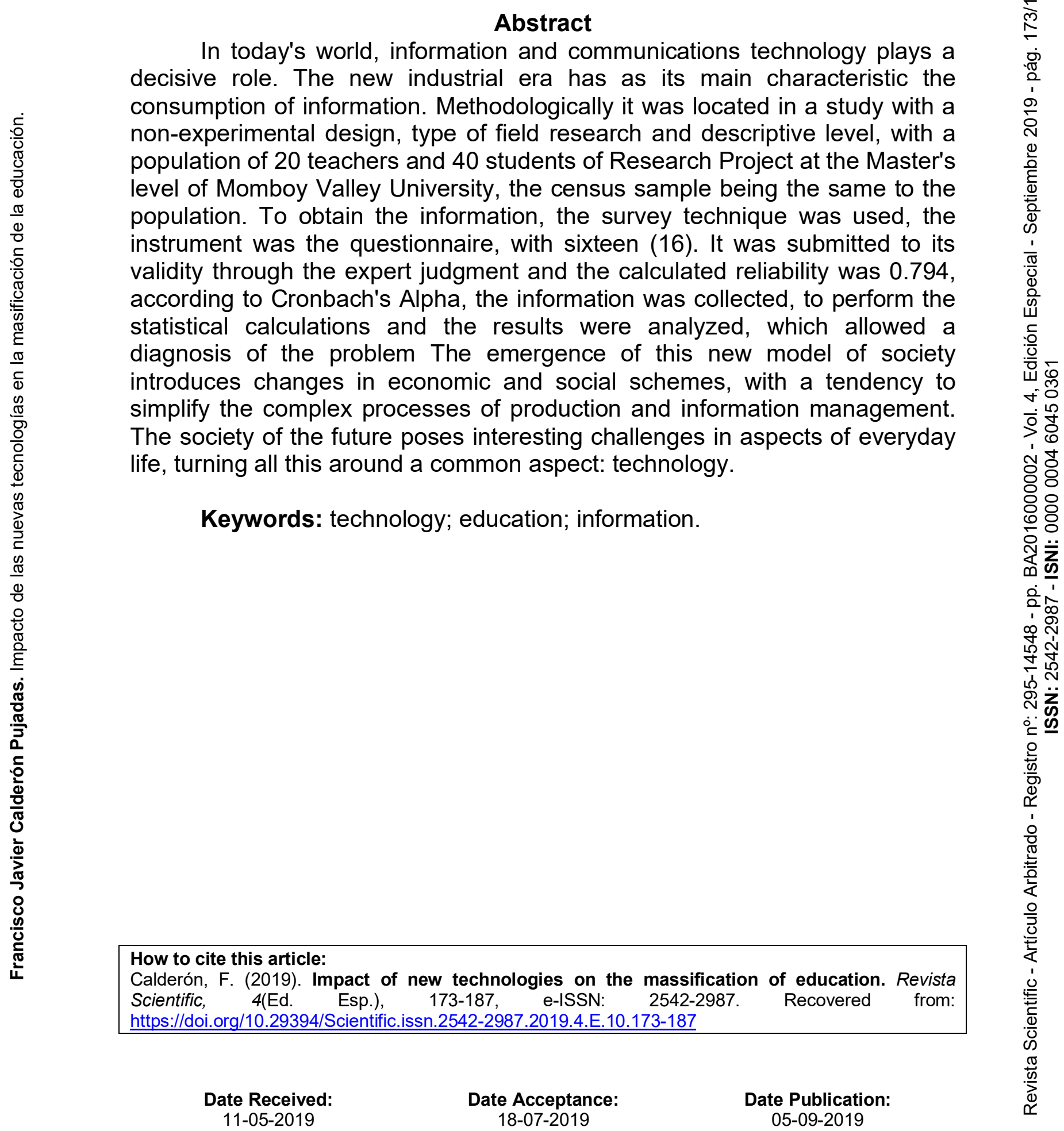




\section{Introducción}

En el mundo de hoy juega un papel determinante la evolución tecnológica para la formación del hombre. A partir de la última revolución tecnológica iniciada a finales del siglo XX, la mayoría de las industrias basan sus esquemas de producción en el desempeño de sistemas comunicacionales.

A diferencia de los esquemas que le han precedido, la nueva era industrial tiene como característica principal el consumo de información en lugar de consumo de materia prima y energía. En el reciente pasado el hombre suministraba y procesaba la información para el desarrollo de tecnologías eficientes que le permitieran reducir su interacción en la obtención de productos de una alta calidad con el menor esfuerzo. El esquema de producción de esta nueva sociedad se basa en el desarrollo de tecnologías para incrementar el procesamiento de la información, obteniendo un alto crecimiento económico con un requerimiento de energía y materias primas limitado.

En este nuevo esquema la economía se sustenta en la información en lugar fuente de energía diferentes como: vapor o electricidad. Razón por la cual, los servicios prestados, está constituido por empresas cuyas actividades se basan en el manejo de datos y del conocimiento cuyo insumo en básicamente de carácter científico. El antiguo modelo industrial está siendo desplazado por un nuevo sistema productivo dominado por este sector.

El surgimiento de este nuevo modelo de sociedad, de acuerdo a Alcalá (2017): "no sólo por su visión sobre la "sociedad red", que actúa como estructura interactiva para la transmisión de la información y del conocimiento, sino también por su discurso académico articulado por la comunicación y los flujos informativos" (pág. 407). La nueva economía se caracteriza por la reducción de las operaciones a nivel empresarial y, por ende, la productividad. Las naciones con alto grado de desarrollo obtienen gran parte de su Producto interno bruto (PIB), como producto de actividades de la industria de la 
información.

Es importante reflexionar sobre el impacto que tendrá en los modelos económicos la creciente demanda de tecnología. La primera y segunda revolución industrial trajeron consigo profundos cambios que aun hoy en día no han sido superados en su totalidad, por lo que se puede inferir que este nuevo cambio industrial repercutirá en los grandes centros económicos, exportando sus consecuencias a los demás países dependientes de la tecnología que estos generan. El crecimiento de la población que se dedica a la industria de la tecnología marcará el rumbo definitivo que seguirá la economía y la generación de empleos del nuevo milenio.

Actualmente, el auge de las tecnologías conduce a una serie de estructuras modificables desde varios puntos de vista, configurando de esta el quehacer cultural, donde el acceso a la información es el vértice principal. ¿Pero estamos aprovechando de verdad todo el potencial que nos brinda las nuevas tecnologías para obtener un verdadero bienestar en este nuevo modelo?

Al pasar los años, se inicia el proceso de transformación en la vida y el quehacer de los hombres, las organizaciones, por esta razón, las diferentes formas de expresar la información a partir del manejo de la tecnología han permitido crear, distribuir y manipular la información como papel esencial para las actividades que se desarrollan en lo social, cultural y económico. De esta manera, la sociedad de la información conduce al manejo de disposiciones históricas que se amalgaman con las transformaciones en cuanto a la mentalidad desde las épocas más antiguas. En cuanto al día a día, la información implica una realidad que se construye con cada actor.

La tecnología educativa como disciplina académica parte en la segunda guerra mundial, para el año de 1946, se implementó como materia de enseñanza en el currículo de la Universidad de Indiana, en el programa de postgrado, bajo la denominación de Educación Audiovisual. La sociedad del 
futuro nos plantea retos interesantes en los diferentes aspectos de la vida.

De allí, que las nuevas tendencias tecnológicas marcaran el camino a seguir en la sociedad del futuro y dependerá del accionar aprovechando el desarrollo de estas tecnologías para el bienestar físico y desarrollo espiritual. Desde finales del siglo XX se están desarrollando y poniendo en práctica tecnologías emergentes que cambiaran radicalmente la manera de pensar y de actuar de nuestros descendientes.

En efecto, los avances tecnológicos han inducido al surgimiento de un nuevo concepto que está impactando a la sociedad, especialmente en todo lo relacionado al mundo de la educación: Las Tecnologías de Información y Comunicación (TIC), delimitadas como puntos de partida, donde tienen vida propia en los espacios educativos, porque han desarrollado herramientas para el aprendizaje significativo vivencial, innovando y enriqueciendo la praxis educativa.

Según Tapscott (2002):

La transformación trae consigo nuevas promesas y oportunidades. Pero también implica riesgos potenciales, tales como invasión de la privacidad, desempleo y hasta convulsión social. El resultado dependerá de la forma como los negocios y la sociedad actúen en respuesta a esta nueva tecnología (pág. 1).

De allí que la dimensión social que alcanzan estas tecnologías obedece a la presión que ejercen en todas las formas de la vida y del trabajo. Entre estos objetivos, la sociedad del nuevo milenio debe plantearse llevar la educación a un mayor número de personas mediante el aprovechamiento de las nuevas tecnologías emergentes, cambiando de esta manera los paradigmas de enseñanza y aprendizaje.

La combinación de varias tecnologías, tales como la comunicación inalámbrica, internet, almacenamiento de datos en la nube, equipos de computación de tamaño compacto (tabletas), sensores inteligentes permitirán 
la implementación de centros de educación sustentables ubicados en sitios remotos donde hasta ahora no había sido posible llevar la educación.

Sin embargo, debemos acotar, tener acceso a grandes volúmenes de información de acuerdo a Zangara (2009):

Este mundo tecnológico, cada vez más complejo, nos desafía a volver, una vez más, sobre las ideas del aprender y del enseñar. Creemos que en ese punto podremos basar una oportuna reflexión acerca de cómo incluir tecnologías en nuestras prácticas de enseñanza. Esta reflexión debería tener un doble sentido: Reflexión epistemológica: Implica pensar acerca de qué son las Tecnologías de la Información y la Comunicación, qué cambios implican en la realidad, para qué sirven, cómo pueden ser utilizadas (en función de la situación educativa, valores éticos, etc.). Reflexión pragmática: Partiendo del conocimiento de estas nuevas tecnologías, se debe analizar cómo es posible potenciar su uso en función de diferentes contextos de enseñanza y aprendizaje (pág. 1).

Para que esta información se convierta en conocimiento es necesario que los sociólogos y docentes especialistas no se queden con las manos cruzadas estudiando y enseñando los contenidos tradicionales; es necesario desarrollar nuevas estrategias, sólo así se desarrollará una sociedad llena innovaciones tecnológicas, para lograr el bien común.

Como reflexión final debemos resaltar que las nuevas tecnologías son solo un medio y somos nosotros, los constructores de esta nueva sociedad, los llamados a revolucionar nuestro estilo de vida haciendo el mejor uso de ellas.

En tal sentido, este complejo y reciente proceso sociopolítico transformacional con sus incertidumbres tecnológicas han tenido una profunda repercusión en la vida de la actual donde se imparte el quehacer educativo, permeando todos los sectores bien definidos de la universidad (docentes, trabajadores administrativos, estudiantes y obrero) y teniendo que escuchar el reclamo de la colectividad social y productiva de acceder a esa tecnología 
dentro del campo laboral y cotidiano.

De esta manera, se deben generar cambios y transformaciones profundas dentro de las instituciones universitarias donde se activen el proceso de construcción y consolidación de nuevas estructuras profesionales que trasciendan el ámbito meramente reivindicativo, en donde esa transformación tenga una alta significación social que atienda las necesidades y de respuestas pertinentes a esa sociedad, generando propuestas y aportes que favorezcan el desarrollo de actitudes y valores.

Asimismo, se debe subsumir esa triada estado-universidad-sociedad donde el estado debe atender y reconocer la universidad en su papel protagónico creadora de conciencia ciudadana y profesionales comprometidos, a través de acciones coherentes que despierten el nivel de conciencia, voluntad de cambio y compromiso de los que hacemos vida activa dentro de estas instituciones. Es nuestra responsabilidad revisar lo que estamos haciendo en la cotidianidad para generar consensos revitalizando el pensamiento crítico e innovador.

Finalmente, la sociedad del conocimiento encauzada por la tecnología induce a las sociedades contemporáneas cuestionando el crecimiento tecnológico TIC's, así como el consumismo y comunicación humana de hoy. Hace fuerte críticas al sistema capitalista y sobre todo a las carencias intelectuales y debilidades de la cultura y educación actual.

En esta sociedad del conocimiento figura una sociedad distinta porque profundiza sus raíces no sólo en la información descomunal sino en el cambio radical igualitario de los pueblos, no propone los clichés del dato sino la transformación del dato y de hombre que lo padece, plantea armonía y la socialización frente al solipsismo individual de los pueblos o entidades humanas, no idiotiza a la persona humana más bien la libera y la hace autónoma y capaz de vivir en bienestar.

En fin, esta transformación pasa por la implantación de ética-política 
(económicas, sociales, culturales, tecnológicas, educativa) que generen cambios radicales de la sociedad.

\section{Metodología}

La investigación se realizó con el propósito de proponer un entorno interactivo virtual de enseñanza y aprendizaje, dirigido al desarrollo de Seminario I, II y III de la Maestría en Gestión de Calidad de la Universidad Miguel de Cervantes (UMC), como parte de la gerencia en el aula para que conduzca al mejoramiento del desempeño del docente, por esta razón, se enmarcó en un modelo cuantitativo.

En lo que respecta a lo expresado por el autor es importante destacar que el método cuantitativo donde se consideran las relaciones causales, busca explicar estas últimas para fundamentar la hipótesis o las interrogantes. Este método utilizado en las investigaciones del contexto social, depende de la aplicación correcta de una serie de pasos, destacándose técnicas para el procesamiento de datos.

El autor de la presente investigación, se sustenta en Palella y Matins (2012): para desarrollar el Diseño, Tipo y Nivel de Investigación del presente trabajo (págs. 23-281); donde se plantea que el Diseño se encuentra bajo la modalidad del Proyecto Factible, el Tipo de Investigación es de Campo y el Nivel de Investigación es Descriptivo y Transeccional.

\section{Resultados}

Para analizar los resultados, se presenta en cada indicador un cuadro de datos, en este cuadro se reflejan las frecuencias absolutas y relativas porcentuales (\%), de cada una de las alternativas, opciones o categorías de respuesta por ítem y su indicador correspondiente.

En el cuadro 1, se plasma lo referente al entorno interactivo virtual, tal como se observa a continuación: 
Cuadro 1. Variable: Entorno Interactivo Virtual. Dimensión: Recursos Tecnológicos. Indicadores: Plataforma de aprendizaje a distancia, ordenadores, sistema operativo.

\begin{tabular}{|c|c|c|c|c|c|c|c|c|c|c|c|}
\hline \multirow{2}{*}{ N. ${ }^{\circ}$} & \multirow{2}{*}{ Ítem } & \multicolumn{2}{|c|}{$\mathbf{S}$} & \multicolumn{2}{|c|}{ Cs } & \multicolumn{2}{|c|}{$\overline{A v}$} & \multicolumn{2}{|c|}{ Cn } & \multicolumn{2}{|c|}{$\mathbf{N}$} \\
\hline & & $\mathrm{fa}$ & $\%$ & fa & $\%$ & fa & $\%$ & fa & $\%$ & fa & $\%$ \\
\hline 1 & $\begin{array}{l}\text { Ha tenido experiencia con } \\
\text { clases en la plataforma de } \\
\text { educación a distancia Moodle. }\end{array}$ & 0 & 0 & 0 & 0 & 8 & 20 & 12 & 30 & 20 & 50 \\
\hline 2 & $\begin{array}{l}\text { Tiene usted acceso a un } \\
\text { computador con Internet fuera } \\
\text { del recinto universitario. }\end{array}$ & 16 & 40 & 12 & 30 & 6 & 15 & 6 & 15 & 0 & 0 \\
\hline 3 & $\begin{array}{l}\text { Utiliza otro sistema operativo } \\
\text { diferente a Windows. }\end{array}$ & 0 & 0 & 0 & 0 & 10 & 25 & 30 & 75 & 0 & 0 \\
\hline
\end{tabular}

Fuente: El Autor (2019).

Gráfico 1. Resultados obtenidos del procesamiento de los ítems 1, 2, y 3.

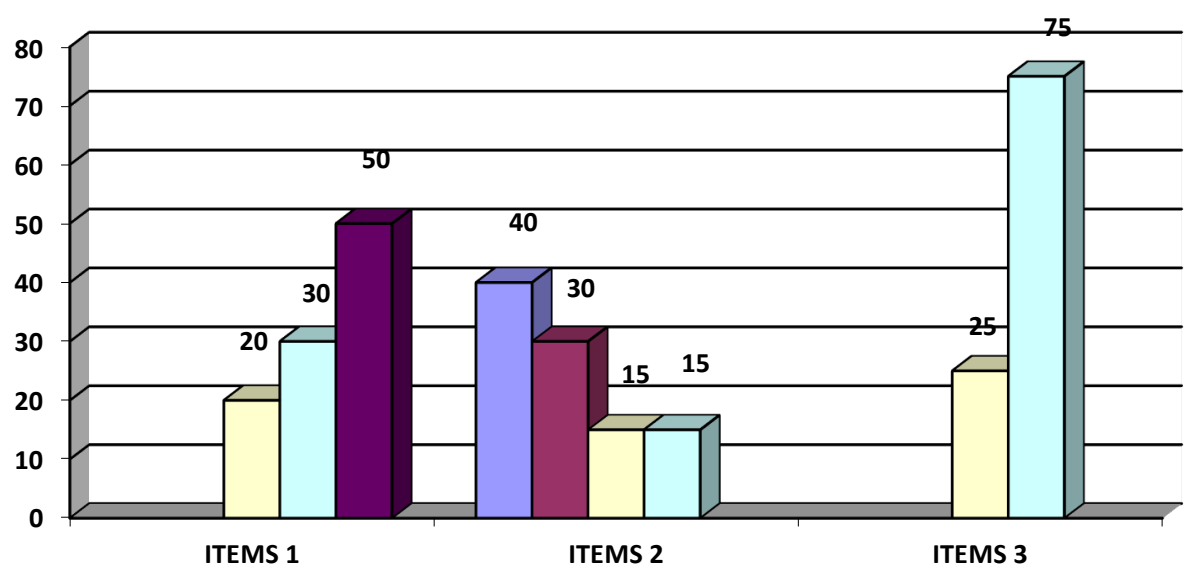

QSIEMPRE ICASI SIEMPRE

DALGUNAS VECES

口CASI NUNCA

aNUNCA

Fuente: El Autor (2019).

En el gráfico 1, con relación al Ítem 1, los sujetos entrevistados respondieron, el cincuenta por ciento $(50 \%)$ Nunca se encuentran familiarizados con la plataforma de educación a distancia Moodle, igualmente 
el treinta por ciento (30\%) Casi Nunca, el veinte por ciento (20\%) Algunas Veces, y por último las opciones Siempre y Casi Siempre no obtuvieron respuesta.

En el ítem 2, el cuarenta por ciento (40\%) Siempre tienen acceso a un computador con Internet fuera del recinto universitario, el Treinta por ciento (30\%) Casi Siempre, la opción Algunas Veces y Casi Nunca representan el quince por ciento (15\%). Nunca no obtuvo respuestas.

Los resultados del ítem 3, son los siguientes: los sujetos encuestados respondieron que el setenta y cinco por ciento (75\%) Casi Nunca, han utilizado diferentes programas en la Asignatura de Proyecto de Investigación en sus clases, así mismo, el veinticinco por ciento (25\%) eligió la opción Nunca.

En la tendencia de la dimensión tecnológica se observa negativa en cuanto a los aspectos que evidencian la poca utilización de diferentes sistemas operativos y la poca familiarización con la plataforma Moodle.

De esta manera las universidades requieren el manejo de diferentes sistemas operativos con el propósito de evolucionar técnicamente proporcionando así diferentes opciones de aprendizaje, con la utilización de los entornos interactivos virtuales en el ámbito educativo. Por otra parte, el uso de software privativo para el proceso de enseñanza a distancia implica un ejemplo ético por parte de la institución educativa y el docente ya que la primera puede adquirir la licencia del programa, pero al momento de distribuirlo entre los alumnos éstas licencias ya no son legales, a menos que el alumno compre el programa por su cuenta.

En cuanto al manejo del entorno interactivo virtual, se visualiza a partir del cuadro 2: 
Cuadro 2. Variable: Entorno Interactivo Virtual. Dimensión: Elementos que integran el EIV. Indicadores: Presentación multimedia, desarrollo de contenidos de EIV, Adaptación de prácticas al entorno virtual.

\begin{tabular}{|c|c|c|c|c|c|c|c|c|c|c|c|}
\hline \multirow{2}{*}{ N. ${ }^{\circ}$} & \multirow{2}{*}{ Ítems } & \multicolumn{2}{|c|}{ s } & \multicolumn{2}{|c|}{ Cs } & \multicolumn{2}{|c|}{$\mathbf{A v}$} & \multicolumn{2}{|c|}{ Cn } & \multicolumn{2}{|c|}{$\mathbf{N}$} \\
\hline & & $\mathrm{fa}$ & $\%$ & fa & $\%$ & fa & $\%$ & fa & $\%$ & fa & $\%$ \\
\hline 4 & $\begin{array}{l}\text { Le gustaría que sus docentes } \\
\text { utilicen contenidos con } \\
\text { diagramación visual resaltante. }\end{array}$ & 36 & 90 & 4 & 10 & 0 & 0 & 0 & 0 & 0 & 0 \\
\hline 5 & $\begin{array}{l}\text { Considera que los recursos } \\
\text { educativos con contenido } \\
\text { multimedia tales como videos, } \\
\text { audio o programas } \\
\text { especializados, complementaría } \\
\text { su aprendizaje. }\end{array}$ & 12 & 30 & 16 & 40 & 8 & 20 & 4 & 10 & 0 & 0 \\
\hline 6 & $\begin{array}{l}\text { Estaría de acuerdo en usar una } \\
\text { herramienta digital que cubra la } \\
\text { carencia del aula virtual en la } \\
\text { Universidad. }\end{array}$ & 14 & 35 & 12 & 30 & 9 & 23 & 5 & 13 & 0 & 0 \\
\hline
\end{tabular}

Fuente: El Autor (2019).

Gráfico 2. Resultados obtenidos al procesar los ítems 4,5 y 6 .

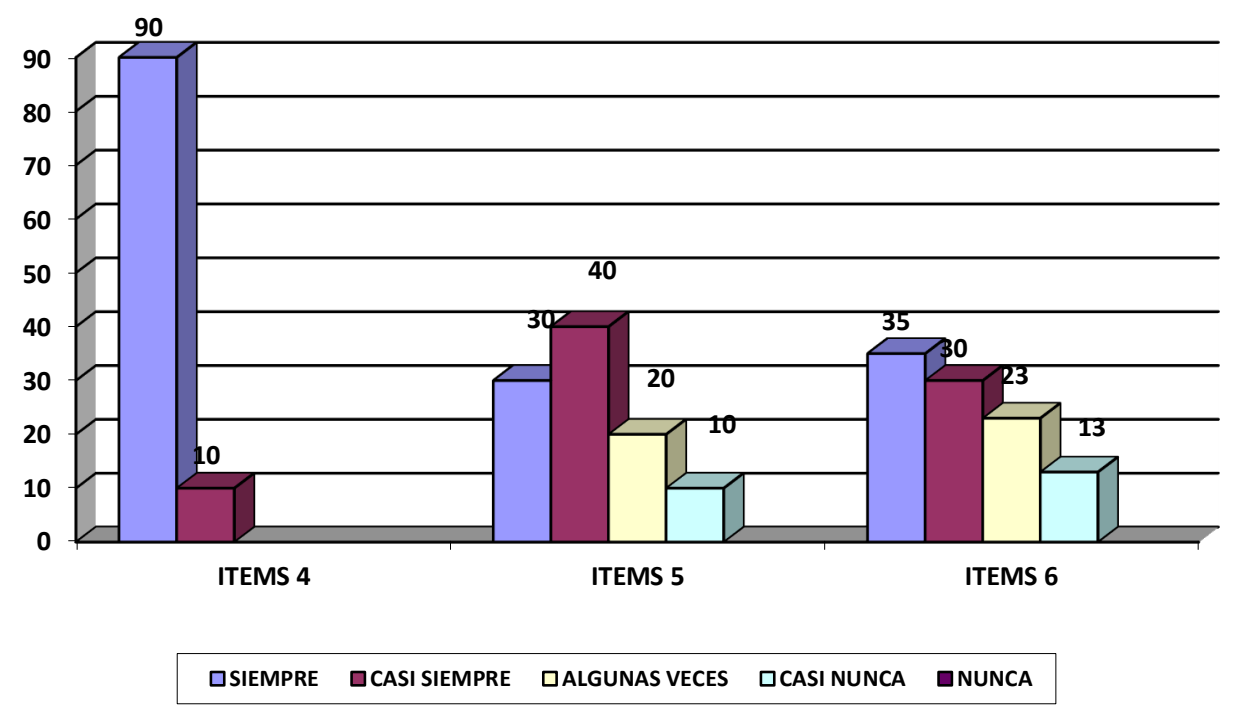

Fuente: El Autor (2019).

En cuanto al gráfico 2, en el ítem 4, se identifica que el noventa por 
ciento $(90 \%)$ de los encuestados respondió Siempre, al indagar sobre si le gustaría que sus docentes utilicen contenidos con diagramación visual resaltante, de igual manera, el diez por ciento $(10 \%)$ de los entrevistados manifestaron Casi Siempre y las opciones Algunas Veces, Casi Nunca y Nunca no obtuvieron respuesta.

En relación a si considera que los recursos educativos con contenido multimedia tales como videos, audio o programas especializados, complementaría su aprendizaje, se observa en el ítem 5 , que al responder se obtuvo el cuarenta por ciento (40\%), optaron por la alternativa Casi Siempre, el treinta por ciento (30\%) respondió Siempre, el veinte por ciento $(20 \%)$ Algunas Veces, el diez por ciento (10\%) Casi Nunca y la opción Nunca no fue seleccionada.

Con respecto al ítem 6, estaría de acuerdo en usar una herramienta digital que cubra la carencia del aula en la Universidad, el treinta y cinco por ciento $(35 \%)$ respondió Siempre, seguido de la opción Casi Siempre con el treinta por ciento $(30 \%)$, veintitrés por ciento $(23 \%)$ para la opción Algunas Veces y trece por ciento (13\%) para la opción Casi Nunca.

De acuerdo con las respuestas proporcionadas se puede determinar que la tendencia es positiva en la dimensión elementos que integran el EIV con respecto a las respuestas aportadas por los estudiantes, con lo cual se corrobora complementar sus estudios a través de herramientas virtuales a distancia. Según Rojano (2003):

Doble reto para los sistemas educativos en los países en desarrollo, pues además de incorporar las TIC a la escuela a través de un uso apropiado para la enseñanza y el aprendizaje, se debe afrontar el hecho de que la mayor parte de los docentes y de los alumnos no posee las competencias informáticas básicas. Concebir modelos de incorporación de las TIC con la tercera concepción implica, junto a ese doble reto, lidiar con las prácticas generadas por un currículo conservador (pág. 138). 
De esta forma, para la creación de entornos de enseñanza y aprendizaje a través de la tecnología de la información existe una amplia gama de herramientas que lo conforman.

\section{Conclusiones}

Los hallazgos derivados de los resultados de la información obtenida a partir de la muestra seleccionada (docentes y alumnos), junto con los aspectos teóricos que sustentaron la investigación, permitieron al investigador deducir las siguientes conclusiones:

Se pudo evidenciar que todos los docentes manifestaron una actitud resistente al cambio en cuanto al uso de la tecnología, plataformas de estudios a distancia, software libre, entre otros, difícilmente logrará convertirse en un buen tutor de un entorno interactivo virtual de enseñanza y aprendizaje, mientras que los estudiantes cuentan con computadores en casa no saben trabajar con la plataforma.

Los estudiantes corroboran el alto porcentaje de aceptación para complementar sus estudios a través de herramientas virtuales a distancia. A su vez, los docentes señalaron que mediante la plataforma, se pueden planificar y desarrollar una serie de actividades las cuales favorecen la comunicación docente-estudiante, existiendo diversas funcionalidades entre las cuales encontramos: el chat que favorece la comunicación de los participantes en tiempo real; además menciona que mediante los foros es donde se dan la mayor parte de los debates, y pueden incluir evaluaciones.

Tanto los estudiantes como los docentes están comprometidos con los adelantos tecnológicos en cuanto a los medios de información y comunicación se refiere, adaptándose al contexto social donde se desarrollan. Es por ello que, es necesario que los docentes en formación involucrados en el proceso escolar son los autores y actores, asimismo, pensar en los usos didácticos que se les darán a las tecnologías, todo esto mediante la organización de nuevos 
enfoques en los procesos de enseñanza y aprendizaje y al perfeccionamiento a nivel de posgrado, para así pasar de una educación estática a una dinámica y cambiante.

\section{Referencias}

Alcalá, M. (2017). La Galaxia Internet: Reflexiones sobre Internet, empresa y sociedad de Manuel Castells. Revista Mexicana de Ciencias Politicas y Sociales, 62(231), 407-412, e-ISSN: 2448-492X. Recuperado de:

http://www.revistas.unam.mx/index.php/rmcpys/article/view/59345

Palella, S., \& Martins, F. (2012). Metodología de la Investigación Cuantitativa. 1ra reimpresión, ISBN: 980-273-445-4. Caracas, Venezuela: Fondo Editorial de la Universidad Pedagógica Experimental Libertador - FEDUPEL.

Rojano, T. (2003). Incorporación de entornos tecnológicos de aprendizaje a la cultura escolar: proyecto de innovación educativa en matemáticas y ciencias en escuelas secundarias públicas de México. Revista Iberoamericana de Educación, (33), 135-165, e-ISSN: 1022-6508. Recuperado de:

https://rieoei.org/historico/documentos/rie33a07.PDF

Tapscott, D. (2002). La economía digital: Promesas y peligros en la era de la inteligencia en redes. Libros de Gerencia Resumidos, Resumido.com. Panamá: Meltom Technologies Inc. Recuperado de: http://www.elmayorportaldegerencia.com/Libros/Economia/[PD]\%20Lib ros\%20-\%20La\%20economia\%20digital.pdf

Zangara, A. (2009). Uso de nuevas tecnologías en la educación: una oportunidad para fortalecer la práctica docente. Puertas Abiertas: Revista de la escuela de lenguas, (5), e-ISSN: 1853-614X. Recuperado de: http://www.puertasabiertas.fahce.unlp.edu.ar/numeros/n5/zangara 
Francisco Javier Calderón Pujadas

e-mail: fcalderon@umcervantes.cl

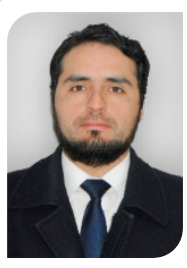

Nacido en Chile, el 14 de octubre del año 1983. Profesor de Educación Básica Mención Matemática; Profesor de la Universidad Miguel de Cervantes (UMC); Se ha desempeñado como Educador de Párvulos en distintos establecimientos educacionales, tanto públicos como privados; Posteriormente, he realizado funciones en instituciones del ámbito educativo y académico; y actualmente como docente en el programa de Magister en Educación de la Universidad Miguel de Cervantes (UMC).

El contenido de este manuscrito se difunde bajo una Licencia de Creative Commons ReconocimientoNoComercial-Compartirlgual 4.0 Internacional 